\title{
RECONSTRUCTING THE KHITAN VOWEL SYSTEM AND VOWEL SPELLING RULE THROUGH THE KHITAN SMALL SCRIPT
}

\author{
MASAMI ŌTAKE \\ Department of Linguistics, Graduate School of Letters, Kyōto University \\ Yoshida-Hommachi, Sakyō-ku, Kyōto, 606-8501 Japan \\ e-mail: masami.t0k1@gmail.com
}

This paper reconstructs the Khitan vowel system by analysing materials concerning the Khitan Small Script. First, the approximate phonetic values of the graphemes were determined by systematically comparing Khitan transcriptions of Chinese words with their original Chinese sounds and by analysing Khitan rhymes. Next, an exhaustive survey of two adjacent graphemes in a corpus elucidated the script's spelling rule and thus were the accurate phonetic values determined. Finally, a comparative study based on the reconstructed values established regular vowel correspondences between Khitan and Mongolian. In conclusion, the author presents twelve Khitan vowel phonemes that are distinguished by four vocalic features.

Key words: Khitan language, Mongolic languages, Khitan script, vowel-overlapping phenomenon, primary long vowel, palatalised vowel, comparative linguistics, decipherment.

\section{Introduction}

In deciphering the Khitan Small Script (henceforth KSS), some materials (such as the ones shown below) are available to determine the phonetic values of the graphemes of KSS:

First, transcriptions of Chinese words in the KSS. Khitan inscriptions contain numerous Chinese words such as Chinese official titles and proper names. A systematic comparison of the Khitan transcriptions of these Chinese words with their original Chinese sounds provides an important clue for determining the phonetic values of KSS.

Second, transcriptions of Khitan words in Chinese characters. Some Chinese literatures like the Liaoshi 遼史 (the History of Liao) and contemporary entombed epitaphs (muzhiming 墓誌銘) written in Chinese contain various Khitan words transcribed 
in Chinese characters. Scholars have compared these Chinese transcriptions with the Khitan notations of the same words and have thereby reconstructed the phonetic values of the graphemes. Examples are given below: ${ }^{1}$

\begin{tabular}{|c|c|c|c|c|}
\hline Chinese & cription & KSS notation & Kane (2009) & Meaning \\
\hline 沙里 & *ša.li & 丈为亲 & $\dot{s}-a-\underline{r} \underline{i}$ & (a male title) \\
\hline 撻不也 & *ta. $\overline{b u} . y \ddot{a}$ & 令生百炎 & $t(e)-\underline{b u}-e i-e r$ & (a person's name) \\
\hline 捏褐 & *nyä.xe & 伏丙 & $\dot{n}(i)-q o$ & 'dog' \\
\hline
\end{tabular}

Such reconstructions, however, are quite problematic, because Chinese has strong restrictions on phonotactics, especially on codas. As a result, the Chinese transcriptions may contain additional vowels to transcribe Khitan codas such as $* l,{ }^{*} b,{ }^{*} x$ that are not allowed in Chinese. Chinese transcriptions therefore do not assure the accuracy of the reconstructed values.

Third, cognates with the Mongolian languages. Scholars have also compared Khitan notations of Khitan words with their cognates in its family languages (i.e. the Mongolian languages) to reconstruct grapheme phonetic values. See the examples below:

\begin{tabular}{|c|c|c|c|c|}
\hline Middle Mongolian & Modern Mongolian & KSS notation & Kane (2009) & Meaning \\
\hline söni & $\check{s} \theta n \sim s \theta n$ & 午杏 & $s-\underline{u n i}$ & 'night' \\
\hline mor $\underline{i}$ & $m \ddot{r}$ & 不代 & $\overline{m-\underline{r i}}$ & 'horse' \\
\hline basa & bas & 丹冬 & $b-\underline{a s}$ & 'again, also' \\
\hline e'ülēn & uul & 土童 & $e u-\underline{u l}$ & 'cloud' \\
\hline
\end{tabular}

These reconstructions are also problematic in that they postulate both preservation and loss of short vowels in non-initial syllables in Khitan without considering sound changes. Since we do not know what changes Khitan has undergone, we cannot determine accurate values by comparing Khitan notations with their cognates.

Forth, rhymes in Khitan inscriptions. Like Chinese ones, Khitan entombed epitaphs have a section written in verse, which is introduced with a phrase equivalent to the Chinese "ming yue 銘曰" ('The inscription says'). An analysis of rhyming words confirms which graphemes share the same or similar segments in phonetic values.

Lastly, orthographic restrictions in the KSS. If a KSS spelling rule is elucidated, it may help determine the phonetic values of the graphemes.

In this paper, I reconstructed the Khitan vowel system by analysing the KSS. As stated above, the second and the third source materials are somewhat problematic for the purpose of determining phonetic values. Hence, I determined the values by using exclusively the first, the fourth, and the last source materials. By using cognates, I then established regular vowel correspondences between Khitan and Mongolian. Based on this, I will reconstruct the Khitan vowel system as shown in Table 1. (2009).

\footnotetext{
${ }^{1}$ Here the phonetic values reconstructed by previous studies are represented by Kane's
} 
Table 1

\begin{tabular}{lcccc}
\hline & \multicolumn{2}{c}{ Front } & \multicolumn{2}{c}{ Back } \\
& Unrounded & Rounded & Unrounded & Rounded \\
\hline Close & $i[\mathrm{i}]$ & $\ddot{u}[\mathrm{y}]$ & $(l[\mathrm{i}])$ & $u[\mathrm{u} \sim \mathrm{u}]$ \\
Near-close & & & & $\sigma[\mathrm{\sigma}]$ \\
Mid & $\ddot{e}[\mathrm{e}]$ & $\ddot{\theta}[\varnothing]$ & $e[\partial \sim \gamma]$ & $\theta[\mathrm{o} \sim \mathrm{\theta}]$ \\
Open & $\ddot{a}[\varepsilon]$ & $\ddot{o}[œ]$ & $a[\mathrm{a}]$ & $o[\mathrm{o}]$ \\
\hline
\end{tabular}

\section{Correspondence to Chinese Sounds}

First, we have no material on Northern Chinese during the Liao period (916-1125) to know its phonology systematically. I thus tentatively refer to Yuan Chinese phonology (13th-14th centuries) which is mainly reconstructed from the rhyme books Zhongyuan yinyun 中原音韻 (henceforth $\mathrm{ZY}$ ) and the Menggu ziyun 蒙古字韻 (henceforth MZ). ${ }^{2}$

The results below ensue from comparing Khitan transcriptions of Chinese words with their Yuan Chinese forms. We found three types of graphemes: vowel graphemes, consonant + vowel graphemes, and vowel + consonant graphemes. ${ }^{3}$ It should be noted that comparisons with Chinese sounds only revealed the grapheme types and their approximate phonetic values.

\subsection{Vowel Graphemes}

Table 2 shows the vowel graphemes ( $\mathrm{V}$ graphemes) that correspond to Yuan Chinese $(\mathrm{M}) \mathrm{Vs}$ in $(\mathrm{I})(\mathrm{M}) \mathrm{V}$ syllables or $(\mathrm{I})(\mathrm{M}) \mathrm{VE}$ syllables $(\mathrm{I}=$ initial, $\mathrm{M}=$ medial, $\mathrm{V}=$ vowel, $\mathrm{E}=$ ending). I assumed that Khitan did not allow rising diphthongs like [ia] and thus Chinese MVs were adapted for monophthongs in Khitan. This assumption is supported by other evidence which will be presented in Section 4.2.

${ }^{2}$ This paper employs the author's notational system of ZY. Note that $p, t, c, \check{c}, k$ represent aspirated $\left[\mathrm{p}^{\mathrm{h}}, \mathrm{t}^{\mathrm{h}}, \mathrm{ts}^{\mathrm{h}}, \mathrm{t}^{\mathrm{h}}, \mathrm{k}^{\mathrm{h}}\right]$ while $b, d, z, \breve{J}, g$ represent unaspirated $\left[\mathrm{p}, \mathrm{t}, \mathrm{ts}, \mathrm{t} \int, \mathrm{k}\right] ; v, \check{z}$ represent approximants; $\ddot{a}, e, l, o, \hat{o}, \ddot{u}$ represent $[\varepsilon, \partial \sim \gamma, \dot{\mathrm{i}}, \mathrm{\jmath}, \mathrm{o}, \mathrm{y}]$.

Previous studies like Shen $(2006,2007)$ have shown that the phonological characteristics of Liao Chinese are similar to those of Yuan Chinese, although some archaic characteristics have been preserved.

${ }^{3}$ Beside these three types, we also found consonant + vowel + consonant graphemes, e.g. 介 $=$ 后 $\mathrm{ZY} * x e w$, 主 $=$ 皇 $\mathrm{ZY} * x w a \eta$. 
Table $2^{4}$

\begin{tabular}{|c|c|c|c|c|c|}
\hline \multicolumn{2}{|c|}{ Grapheme } & ZY final & Example & ZY final & Example \\
\hline 为 $_{189}$ & 〈aa〉 & 家麻 * $-a$ & 不为馬 $* m \underline{a}$ & $\begin{array}{l}\text { 寒山 *-an } \\
\text { 監戌*-am }\end{array}$ & 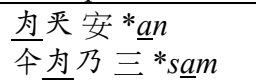 \\
\hline 斗 335 & 〈ää〉 & 家麻 *-ya & 几斗家* $g y a$ & $\begin{array}{l}\text { 寒山*-yan } \\
\text { 監咸*-yam }\end{array}$ & 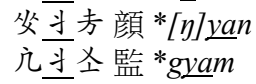 \\
\hline 炎 $_{348}$ & $\langle e e\rangle$ & 歌戈 $*_{-} e$ & 几炎 哥 * $g \underline{e}$ & & \\
\hline 交 $_{327}$ & 〈ëë〉 & 車遮 *-yä & 平交姐 $*_{z y \ddot{a}}$ & $\begin{array}{l}\text { 先天 *-yän } \\
\text { 廉纖 *-yäm }\end{array}$ & 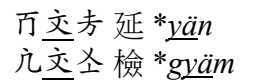 \\
\hline 及 $_{186}$ & $\langle 00\rangle$ & 歌戈 *-wô & 伞及左 $*_{z} \underline{w o}$ & 桓歡 *-won & 化及尔亂*lwon \\
\hline 艾 082 & 〈öö〉 & & & 先天 *- $\overline{y \ddot{a} n}$ & 灸交吅 元 $* \overline{[\eta]} y \ddot{a} n$ \\
\hline 伴 226 & $\langle\ddot{\theta} \ddot{\theta}\rangle$ & & & 先天 *-ÿ̈n & 灸伴会元 * $[\eta] \underline{u a ̈} n$ \\
\hline 及 131 & $\langle\mathrm{uu}\rangle$ & 魚模 *-u & 公互奴 * $n \underline{u}$ & & \\
\hline 尒 245 & $\langle\mho \mho\rangle$ & 魚模 *-u & 半全祖 $* z \underline{u}$ & & \\
\hline 岀 289 & 〈üǜ & 魚模*-ü & 岀禹 * $\underline{\ddot{u}}$ & & \\
\hline 兴 339 & 〈ii〉 & 齊微 $*_{-} i$ & 兴夷 $*_{i}$ & & \\
\hline 合 353 & $\langle 11\rangle$ & 支思 *-l & 半谷子 $*_{z \underline{l}}$ & & \\
\hline
\end{tabular}

\subsection{Consonant + Vowel Graphemes}

Table 3 shows the consonant + vowel graphemes (CV graphemes) that correspond to Yuan Chinese I(M)Vs in I(M)V syllables or I(M)VE syllables.

Table 3

\begin{tabular}{|c|c|c|c|}
\hline \multirow{2}{*}{$\begin{array}{l}\text { Group } \\
\langle\text { Caa〉 }\end{array}$} & \multicolumn{2}{|c|}{ Grapheme } & \multirow{2}{*}{$\frac{\text { ZY form }}{\text { 王八*ba }}$} \\
\hline & $z_{160}$ & 〈baa & \\
\hline & 南 375 & 〈čaa〉 & 南察*ča \\
\hline & 为 003 & $\left\langle\mathrm{x}^{\mathrm{w}} \mathrm{a} a\right\rangle$ & 丙化 *xwa \\
\hline & 何 229 & $\langle$ taa $\rangle$ & 仍乎 檀 $* t a n$ \\
\hline & 分 214 & $\langle$ daa $\rangle$ & 公中大 * day \\
\hline & 力 $_{139}$ & $\langle$ naa $\rangle$ & 力乃男 * \\
\hline & $子_{150}$ & 〈ॅ̆aа & 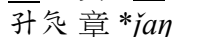 \\
\hline & 巫 053 & 〈xaa〉 & 巫乎韓 * $x a n$ \\
\hline 〈Cää〉 & 不 ${ }_{130}$ & 〈xää〉 & 不下*xya \\
\hline$\langle\mathrm{Cee}\rangle$ & 汉 283 & $\langle$ kee $\rangle$ & 汉可*ke \\
\hline$\langle\mathrm{CoO}\rangle$ & 再 017 & $\langle$ doo〉 & 再多 $* d w o$ \\
\hline & 尖 057 & $\langle$ xoo $\rangle$ & 秃 和 *xwô \\
\hline & $4_{213}$ & $\langle$ too $\rangle$ & 生尔團 *twon \\
\hline & 尚 298 & $\langle$ soo〉 & 尚尔攅 $*$ cwon \\
\hline
\end{tabular}

\begin{tabular}{|c|c|c|}
\hline Group & Grapheme & ZY form \\
\hline \multirow[t]{9}{*}{$\langle\mathrm{Cuu}\rangle$} & 㕣 $241\langle$ puu $\rangle$ & 分府 * $f u$ \\
\hline & 少 $358\langle\mathrm{muu}\rangle$ & 半墓 ${ }^{*} m u$ \\
\hline & 贫 202 〈tuu〉 & 贫徒 * $t u$ \\
\hline & 们 237 〈duu〉 & 们都 $* d u$ \\
\hline & 发 208 〈luu〉 & 发盧 *lu \\
\hline & 伅 $233\langle\mathrm{kuu}\rangle$ & 伅哭 * $k u$ \\
\hline & 北 036 〈xuu〉 & 北虎 *xu \\
\hline & 马 $_{147}$ 〈ॅuu〉 & 马火 中 * ${ }^{\prime} \underline{u} \eta^{w}$ \\
\hline & $几_{178}\langle\mathrm{kuu}\rangle$ & 几火孔*ku的 \\
\hline \multirow[t]{3}{*}{ 〈Cii〉 } & 付 225 〈bii〉 & 付畢 $* b i$ \\
\hline & 主 037 〈tii〉 & 主帝 $* d i$ \\
\hline & 友 087 〈jii〉 & 友知 $*$ ju \\
\hline \multirow[t]{3}{*}{$\langle\mathrm{C} 11\rangle$} & 进 083 〈S11〉 & 进司 $*_{s l}$ \\
\hline & 5 & 与旨 $* j l$ \\
\hline & 北 & 北使 * ${ }^{\prime s} l$ \\
\hline
\end{tabular}

${ }^{4}$ The subscript number after a KSS grapheme indicates the grapheme number assigned by Qingge'ertai et al. (1985). The value in angle brackets « > refers to the phonetic value that is ultimately proposed in this paper. 


\subsection{Vowel + Consonant Graphemes}

Table 4 shows the vowel + consonant graphemes (VC graphemes) that correspond to Yuan Chinese (M)VEs in (I)(M)VE syllables. We also found other VC graphemes which correspond to Middle Chinese (M)VEs in rusheng 入聲 syllables (see Table 5). ${ }^{5}$ Note that Khitan inscriptions reflect two Chinese varieties in respect of rusheng syllables: one preserved Middle Chinese rusheng codas ${ }^{*}-p, *_{-} t, *_{-} k, *_{-} k^{w}$ as $-b,-r,-g,-g^{w}$ and the other lost them like Yuan Chinese. These two varieties are used together in inscriptions.

\section{Table 4}

\begin{tabular}{|c|c|c|}
\hline Grapheme & ZY final & Example \\
\hline 中 $_{122}\langle\mathrm{ay}\rangle$ & 皆來 *-ay & 八中開 $* k a y$ \\
\hline 禾 $_{197}$ 〈äy〉 & 皆來 *-yay & 八年客 *kyay \\
\hline 太 $_{107}\langle\mathrm{oy}\rangle$ & 皆來 *-way & 莫帥 * \\
\hline $\bar{乃}_{020}\langle\mathrm{ey}\rangle$ & 齊微 *-ey & 坌页德 * dey \\
\hline 众 $262\langle u y\rangle$ & 齊微 *-uе̌y & 公夾內 $* n u \underline{u} y$ \\
\hline 乎 $_{011}\langle\mathrm{an}\rangle$ & 寒山*-an & 为乎安 * $\underline{a n}$ \\
\hline 方 $_{073\langle a ̈ n 〉}$ & 寒山 *-yan & 灸斗去顔 *[n]yan \\
\hline & 先天 *-yän & 百交方延 * yän \\
\hline 吅 324 〈Ön〉 & 先天 *-yän & 众文品宣 *syän \\
\hline 公 251 〈een〉 & $\begin{array}{l}\text { 先天 *-yän } \\
\text { 桓歡 *-won }\end{array}$ & $\begin{array}{l}\text { 伴会 院 * }{ }^{y a ̈ n} \\
\text { 曲公 觀 * } g \underline{w o n}\end{array}$ \\
\hline 小 $_{154}$ 〈on〉 & 桓歡 *-won & 生尔團 *twon \\
\hline 本 $140 〈 \mathrm{en}$ 〉 & 真文*-en & 不市門 * mue \\
\hline 雨 $018\langle$ in & 真文 *-iěn & 伞雨進 *ziěn \\
\hline 少273〈un〉 & 真文 *-uĕn & 爸憂敦 *duĕn \\
\hline 亦 $329\langle\ddot{u}$ 〉 & 真文 *-üĕn & 亢亦軍 * güüen \\
\hline$火_{345}\left\langle u^{w}{ }^{w}\right\rangle$ & 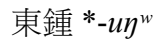 & 几炎公 * $g \underline{u \eta^{w}}$ \\
\hline 半 $357\left\langle 0 \eta^{\mathrm{w}}\right\rangle$ & 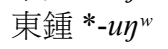 & 平坐宗 $*_{z u \eta^{w}}$ \\
\hline 凡 $_{181\left\langle\ddot{u} \eta^{\mathrm{w}}\right\rangle}$ & 東鍾 *-ü $\eta^{w}$ & 坐风龍 $* l \underline{\ddot{u} \eta^{w}}$ \\
\hline
\end{tabular}

\begin{tabular}{|c|c|c|}
\hline Grapheme & ZY final & Example \\
\hline 在 $161\langle\mathrm{aw}\rangle$ & 蕭豪 $*-a w$ & 几龶高 * $g a w$ \\
\hline \multirow[t]{2}{*}{ 号 $362\langle a ̈ w\rangle$} & 蕭豪 *-yaw & 几步校*gyaw \\
\hline & 蕭豪*-yäw & 午步小*syäw \\
\hline 土 $_{067}\langle\mathrm{ew}\rangle$ & 尤侯 *-ew & 几土狗 *gew \\
\hline 丙 $019\langle\ddot{e} w\rangle$ & 尤侯 *-iěw & 夾丙留*liěw \\
\hline$乃_{184\langle\mathrm{am} 〉}$ & 監咸 *-am & 力乃男*nam \\
\hline \multirow[t]{2}{*}{ X $_{270}$ 〈äm〉 } & 監咸*-yam & 几斗企監 *gyam \\
\hline & 廉纖*-yäm & 几企檢 *gyäm \\
\hline 企 257 & 侵尋 *-em & 丈企潘 *šiěm \\
\hline 不 ${ }_{133}\langle\mathrm{im}\rangle$ & 侵尋 *-iěm & 几㕛金*giěm \\
\hline 志 $072\langle$ üm〉 & 真文*-iĕn & 业克品 * pién ${ }^{6}$ \\
\hline 気 $199\langle a y\rangle$ & 江陽 *-ay & 夾氛郎*lan \\
\hline 〈äy〉 & 江陽 *-yay & 半乎將 $* z y a \eta$ \\
\hline 黑 $071\langle 0 \mid\rangle$ & 江陽 *-way & 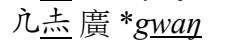 \\
\hline \multirow[t]{2}{*}{ 亚062〈ëy〉 } & 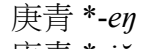 & 丈亚省*šen \\
\hline & 青 *-iěy & 几亚 耿 * g $^{i \underline{i e \eta}}$ \\
\hline & 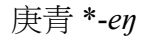 & 伞灸僧 * sen \\
\hline & 庚青 *-iěy & 八同興 *xiěy \\
\hline
\end{tabular}

${ }^{5}$ This paper uses the author's notational system of Late Middle Chinese (LMC). Note that $a, \dot{a}, \ddot{a}, e, o, \hat{o}, \ddot{u}$ represent $[\mathrm{a}, \mathrm{a}, \varepsilon, \partial \sim \gamma, \mathrm{\rho}, \mathrm{o}, \mathrm{y}] ; \dot{s}, s+$ represent $[\mathrm{c}, \mathrm{s}]$.

6 品 (LMC * $p^{\prime}$ iěm, MZ p ${ }^{\prime}$ im) is also transcribed as 业㞋 in the KSS, therefore the dissimilation of labial coda $*-m>-n$ has not yet occurred in Liao Chinese.

${ }^{7}$ In the KSS, Middle Chinese division-II syllables of the geng 梗 rhyme class (e.g. 省 LMC *sán and 耿 LMC *kän) have not yet merged into the zeng 曾 rhyme class (Shen 2006, p. 495). These syllables are transcribed with 亚 (062) which is not used for the zeng rhyme class. 
Table 5

\begin{tabular}{|c|c|c|c|}
\hline \multicolumn{2}{|c|}{ Grapheme } & \multirow{2}{*}{$\begin{array}{l}\text { LMC final } \\
\text { 咸*-ap }\end{array}$} & \multirow{2}{*}{$\begin{array}{l}\text { Example } \\
\text { 卅为玍臘 } \mathrm{ZY} * l a<\mathrm{LMC} * \text { lap }\end{array}$} \\
\hline 玍 196 & $\langle a b\rangle$ & & \\
\hline 丹 $_{311}$ & $\langle e b\rangle$ & 深*-ep & 丈再十 $\mathrm{ZY} * \check{s} i<\mathrm{LMC} *{ }^{*} i \underline{\underline{e} p}$ \\
\hline 无 $_{127}$ & $\langle\mathrm{ib}\rangle$ & 深*-iepp & 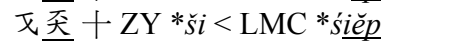 \\
\hline$刃_{137}$ & 〈ür〉 & 臻*-üĕt & 又忍密 $\mathrm{ZY} *$ * muёj < LMC *müĕt \\
\hline 勺 165 & 〈ig〉 & 梗*-yäk & 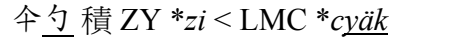 \\
\hline 久 172 & $\left\langle u^{\mathrm{w}}\right\rangle$ & 通 $*-\hat{o} k^{w}$ & 夾全祿 $\mathrm{ZY} * l u<\mathrm{LMC} * l \underline{\hat{o} k^{w}}$ \\
\hline 非 $_{282}$ & $\left\langle u_{\text {g̈w }}\right\rangle$ & 通*-yok ${ }^{w}$ & 少岀非玉 $\mathrm{ZY} *[\eta] \ddot{u}<\mathrm{LMC} * \eta y o k^{w}$ \\
\hline 用 $_{138}^{20}$ & $\left\langle\mathrm{ewg}^{\mathrm{w}}\right\rangle$ & 通*-ewk $k^{w}$ & 更叔 $\mathrm{ZY} * s ̌ u ̈<\mathrm{LMC} *$ syewk \\
\hline
\end{tabular}

\section{Analysis of Rhyming Words}

In Khitan rhymes, each rhyming line usually consists of four words; the last words in the even lines (and sometimes also in the first line) rhyme with each other. ${ }^{8}$

Table 6 shows extracts of rhyming monosyllabic open-syllable words from Khitan rhymes. They are divided into five groups and are presumed to share the same, or nearly the same, vowel in each group.

Table 6

\begin{tabular}{|c|c|c|}
\hline Group & Rhyming words & Source \\
\hline $\mathrm{Caa}$ & $\begin{array}{l}\text { 力 } n a a \text {, 何 } t a a \text {, 几为 } g a a, \text { 六 } d a a \text {. } \\
\text { 巫 } x a a \text {, 王 } b a a \text {. }\end{array}$ & $\begin{array}{l}\text { Daozong } 28-29 \\
\text { Xingzong } 31-32\end{array}$ \\
\hline Cä̈̈ & 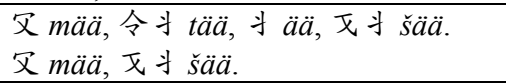 & $\begin{array}{l}\text { Daozong } 14-15 \\
\text { Xuanyi } 19\end{array}$ \\
\hline Cёё & 公交 $n \ddot{e} \ddot{e}$, 丹交 $b \ddot{e} e$, 万交 yëe. & Renyi 23-24 \\
\hline $\mathrm{Coo}$ & 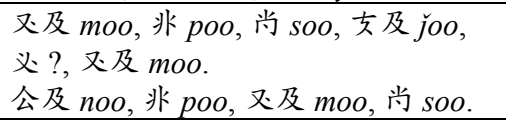 & $\begin{array}{l}\text { Daozong } 21-22 \\
\text { Tadii } 23-24\end{array}$ \\
\hline $\begin{array}{l}\text { Cuu } \\
/ \mathrm{C} v \mho\end{array}$ & 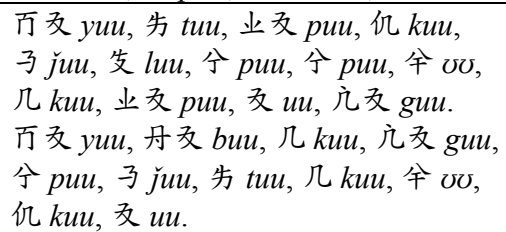 & Mt. Haitang 11-13 \\
\hline
\end{tabular}

${ }^{8}$ Shen (2009) is the first systematic study on Khitan rhymes. He mistakenly assumed that only the last grapheme in a word is involved in rhyming. In fact, the whole word is involved. For

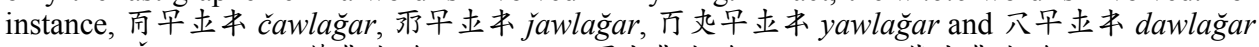
rhyme in Čawj 26-27; 纺化㕕炎 čewdeğeer, 而土化炎炎 yewdeğeer, 伏土化㕕炎 ňewdeğeer and 土化炎炎 ewdegeer rhyme in Daozong 13; 圠元 bideg, 古勺 idig, 火古勺 kidig and 令克勺 tidig rhyme in Hongben 17-18. 
To summarise the observations made in Sections 2 and 3, the presumed V graphemes and CV graphemes are shown in Table 7.

Table 7

\begin{tabular}{|c|c|c|}
\hline & V graphemes & CV graphemes \\
\hline$a$ & 为 $\langle a a\rangle$ & 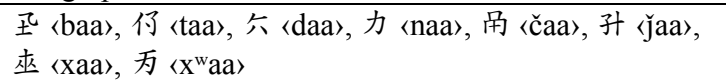 \\
\hline$\ddot{a}$ & 斗〈ää〉 & 又〈mää〉, 不〈xää〉 \\
\hline$e$ & 炎 〈ee〉 & 汉〈kee〉 \\
\hline$\ddot{e}$ & 交〈ëë〉 & \\
\hline$o$ & 及〈0o〉 & 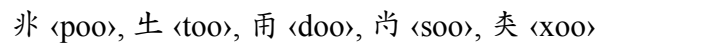 \\
\hline$\ddot{o}$ & 艾〈öö〉 & \\
\hline$\ddot{\theta}$ & 伴〈尚〉 & \\
\hline$u / v$ & 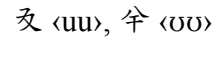 & 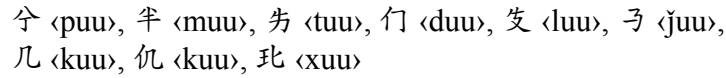 \\
\hline$\ddot{u}$ & 岀〈üü〉 & \\
\hline$i$ & 兴〈ii〉 & 付〈bii〉, 主〈tii〉, 友〈jiì \\
\hline$l$ & 吕 〈11〉 & 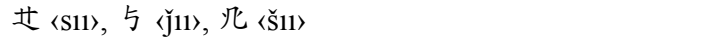 \\
\hline
\end{tabular}

\section{Analysis of the Khitan Spelling Rule for Vowels}

In the transcriptions of Chinese words, a vowel-overlapping phenomenon is observed:

\begin{tabular}{|c|c|c|c|}
\hline$x$ & 〈(e)g-ää-äm〉 & 監 $(Z Y *$ gyam $)$ & $\langle(\mathrm{V}) \mathrm{C}-\mathrm{V}-\mathrm{VC}\rangle$ \\
\hline 南 & 〈čaa-ạ〉〉 & 長 $(Z \mathrm{Y} * c ̌ a \eta)$ & $\langle\mathrm{CV}-\mathrm{VC}\rangle$ \\
\hline 㘯火 & $\left\langle\right.$ tuu-un $\left.{ }^{\mathrm{w}}\right\rangle$ & 同 $\left(\mathrm{ZY} * t u \eta^{w}\right)$ & $\langle\mathrm{CV}-\mathrm{VC}\rangle$ \\
\hline
\end{tabular}

Many researchers refer to this phenomenon, but none of them has successfully generalised it. In this section, I conduct an exhaustive survey of bigrams from a corpus in order to elucidate this vowel-overlapping rule.

\subsection{Method and Results of the Survey}

The question now is that in a sequence of two adjacent graphemes $G_{1}-G_{2}$ within a word, if $\mathrm{G}_{1}$ is a vowel-final grapheme, then what grapheme would the $\mathrm{G}_{2}$ be. To address this question, I counted in my corpus the number of tokens of $\mathrm{G}_{1}-\mathrm{G}_{2}$ bigrams where $\mathrm{G}_{1}$ is any of the $\mathrm{V}$ and $\mathrm{CV}$ graphemes listed in Table $7 .^{9}$ The corpus consisted

${ }^{9}$ I exclude 伊 〈ë̈̈〉 and the four graphemes representing 〈(C)11〉 from the survey because of the former's limited quantity and the latter's characteristic of exclusive use for Chinese loanwords. 
of 30 inscriptions that are equivalent to approximately three quarters of the existing KSS inscriptions. ${ }^{10}$

Table 8 shows the results of the survey. ${ }^{11}$

\subsection{Abductive Inference of a Rule}

From these data, the following generalisation can be made:

(i) In principle, $\mathrm{a}_{2}$ that can follow a $(\mathrm{C}) \mathrm{V}$ grapheme whose vowel is $\mathrm{V}_{i}$ does not follow the other $(\mathrm{C}) \mathrm{V}$ graphemes whose vowels are not $\mathrm{V}_{i}$.

${ }^{10}$ The following list contains the inscriptions included in my corpus:

[1] Epitaph of Čawn̆ Lüü-peň (Yelü Zongjiao 耶律宗教). Dated 1053, 36 lines.

[2] Broken Epitaph of Gawň Puu-lëw (Xiao Shenhui 蕭慎徽). Dated 1057, 32 lines.

[3] Epitaph of Puиnuиn̆ Toxoser. Dated 1068, 26 lines.

[4] Epitaph of Ujeeň (Yelü Jue 耶律玦). Dated 1071, 46 lines.

[5] Epitaph of Onun̆ Gaw-šib. Dated from 1076 onward, 26 lines.

[6] Epitaph of Kuyreğeen̆ Xaadii. Dated 1080, 32-line text and 14-word title.

[7] Epitaph of Bedelbeň Čaw̌. Dated 1082, 28-line text and 15-word title.

[8] Broken Epitaph of Yuyuñ. Dated 1088, 43 lines.

[9] Epitaph of Teleğeen̆ Xodvxvй. Dated 1091, 39 lines.

[10] Epitaph of Lëënen̆ Xaar. Dated 1091, 48 lines.

[11] Epitaph of Sarağan̆ Dileed. Dated 1092, 41 lines.

[12] Epitaph of Orelbeň Yëerruu (Yelü Zhixian 耶律智先). Dated 1094, 27 lines.

[13] Epitaph of Guyneň Nuu. Dated 1099, 48 lines.

[14] Epitaph of Awloğooň Uyeer (Yelü Hongyong 耶律弘用). Dated 1100, 32 lines.

[15] Epitaph of Saraan̆ Šiluu. Dated 1100, 13-line text and 7-word title.

[16] Epitaph of Kuипun̆ Dilee (Yelü Chengkui 耶律承窺). Dated 1101, 34 lines.

[17] Eulogy for Emperor Daozong 道宗. Dated 1101,37-line text and 14-word title.

[18] Eulogy for Empress Xuanyi 宣懿. Dated 1101, 30-line text and 7-word title.

[19] Epitaph of ठ̋rdoğoon̆ Umur. Dated 1102, 51 lines.

[20] Epitaph of Šiluuñ J̌uurjee (Xiao Zhiwei 蕭知微). Dated 1107, 29 lines.

[21] Broken Epitaph from Nangou 南溝, Sanshan township 三山鄉. Dated 1108, 26 lines.

[22] Eulogy for Imperial Grand Uncle Yihe renshou 義和仁壽皇太叔祖 (Yelü Hongben 耶律弘本). Dated 1110, 25-line text and 6-word title.

[23] Epitaph of Uuruğweě̆, Wife of Yelü Hongben. Dated 1110, 24-line text and 8-word title.

[24] Epitaph of Baysbeň Čalaa (Xiao Min 蕭旼). Dated 1113, 27 lines.

[25] Epitaph of Ärluğoon̆ Dilugw. Dated 1114, 51 lines.

[26] Epitaph of Tadii, Wife of Xiao Xiaosi 蕭孝思. Dated 1115, 25 lines.

[27] Record of the Journey of the Imperial Brother of the Great Jin Dynasty 大金皇弟都 統經略郎君行記. Dated 1134, 5 lines.

[28] Epitaph of Oryeeň J̌uurjee (Xiao Zhonggong 蕭仲恭). Dated 1150, 50-line text and 9-word title.

[29] Broken Epitaph from Yemaotai 葉茂臺. Undated, 6 lines.

[30] Broken Epitaph from Mount Haitang 海棠山. Undated, 13 lines.

${ }^{11}$ I did not count bigrams that included (an) illegible or fully lost grapheme(s). Due to space limitations, I omitted the individual number of each CV grapheme and showed only the total number of CV graphemes that had a common vowel. 
Table 8

\begin{tabular}{|c|c|c|c|c|c|c|c|c|c|c|c|c|c|c|c|c|c|c|}
\hline \multirow{2}{*}{\multicolumn{3}{|c|}{$\mathrm{G}_{2} \mathrm{G}_{1}$}} & \multicolumn{2}{|c|}{$a a$} & \multicolumn{2}{|c|}{$\ddot{a} \ddot{a}$} & \multirow{2}{*}{$\begin{array}{l}\ddot{e} \ddot{e} \\
\text { 文 }\end{array}$} & \multirow{2}{*}{\begin{tabular}{l|l}
$\ddot{o ̈ o}$ \\
芝
\end{tabular}} & \multicolumn{2}{|c|}{$O O$} & \multicolumn{3}{|c|}{$u u / v v$} & \multirow{2}{*}{$\begin{array}{ll}\text { üü } \\
\text { 岀 } \\
\end{array}$} & \multicolumn{2}{|c|}{$i i$} & \multicolumn{2}{|c|}{$e e$} \\
\hline & & & 为 & $\mathrm{Caa}$ & 斗 & Cää & & & & $\mathrm{Coo}$ & & Cuu & 全 & & & Cii & & Cee \\
\hline \multirow{21}{*}{$a$} & 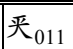 & 〈an〉 & 127 & 39 & 5 & 0 & 0 & 0 & 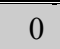 & 0 & 0 & 0 & 0 & 0 & 0 & 0 & 0 & $\overline{0}$ \\
\hline & 平 $_{051}$ & $\langle a \breve{g}\rangle$ & 17 & 210 & 3 & 0 & 0 & 0 & 0 & 0 & 0 & 0 & 0 & 0 & 0 & 0 & 0 & 0 \\
\hline & 共 ${ }_{069}$ & $\left\langle a l^{\prime}\right\rangle$ & 188 & 3 & 0 & 0 & 0 & 0 & 0 & 0 & 0 & 0 & 0 & 0 & 0 & 0 & 0 & 0 \\
\hline & 丂 $_{098}$ & $\langle\mathrm{al}\rangle$ & 165 & 65 & 0 & 0 & 0 & 0 & 0 & 0 & 0 & 0 & 0 & 0 & 0 & 0 & 0 & 0 \\
\hline & 东 099 & $\langle\mathrm{ad}\rangle$ & 78 & 10 & 0 & 0 & 0 & 0 & 0 & 0 & 0 & 0 & 0 & 0 & 0 & 0 & 0 & 0 \\
\hline & 中 $_{122}$ & $\langle a y\rangle$ & 153 & 52 & 1 & 0 & 0 & 0 & 0 & 0 & 0 & 0 & 0 & 0 & 0 & 0 & 0 & 6 \\
\hline & 中 $_{123}$ & $\langle a r\rangle$ & 354 & 42 & 1 & 0 & 0 & 0 & 0 & 0 & 0 & 0 & 0 & 0 & 0 & 0 & 0 & 0 \\
\hline & 刘 ${ }_{151}$ & $\langle\mathrm{ax}\rangle$ & 4 & 4 & 1 & 0 & 0 & 0 & 0 & 0 & 0 & 0 & 0 & 0 & 3 & 1 & 2 & 0 \\
\hline & 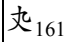 & $\langle\mathbf{a w}\rangle$ & 0 & 3 & 0 & 0 & 0 & 0 & 0 & 0 & 0 & 0 & 0 & 0 & 0 & 0 & 0 & 0 \\
\hline & 冬 ${ }_{174}$ & $\langle$ as $\rangle$ & 15 & 40 & 0 & 0 & 0 & 0 & 0 & 0 & 0 & 0 & 0 & 0 & 0 & 0 & 0 & 0 \\
\hline & $乃_{184}$ & $\langle\mathbf{a m}\rangle$ & 42 & 10 & 3 & 0 & 0 & 0 & 0 & 0 & 0 & 0 & 0 & 0 & 0 & 0 & 0 & 0 \\
\hline & 为 189 & $\langle$ aa〉 & 0 & 5 & 0 & 0 & 0 & 0 & 0 & 0 & 0 & 0 & 0 & 0 & 0 & 0 & 0 & 0 \\
\hline & 生 196 & $\langle\mathbf{a b}\rangle$ & 22 & 7 & 0 & 0 & 0 & 0 & 0 & 0 & 0 & 0 & 0 & 0 & 0 & 0 & 0 & 0 \\
\hline & 氙 199 & $\langle$ an $\rangle$ & 2 & 61 & 0 & 0 & 0 & 0 & 0 & 0 & 0 & 0 & 0 & 0 & 0 & 0 & 0 & 0 \\
\hline & 先 203 & $\langle$ ard $\rangle$ & 0 & 13 & 0 & 0 & 0 & 0 & 0 & 0 & 0 & 0 & 0 & 0 & 0 & 0 & 0 & 0 \\
\hline & X $_{269}$ & $?$ & 1 & 0 & 0 & 0 & 0 & 0 & 0 & 0 & 0 & 0 & 0 & 0 & 0 & 0 & 0 & 0 \\
\hline & 出 290 & $\langle a n ̌\rangle\rangle$ & 332 & 14 & 1 & 0 & 0 & 0 & 0 & 0 & 0 & 0 & 0 & 0 & 0 & 0 & 0 & 0 \\
\hline & 斥 $_{312}$ & $\langle$ ald $\rangle$ & 16 & 25 & 0 & 0 & 0 & 0 & 0 & 0 & 0 & 0 & 0 & 0 & 0 & 0 & 0 & 0 \\
\hline & 共__ & $\langle a \bar{\jmath}\rangle$ & 59 & 16 & 0 & 0 & 0 & 0 & 0 & 0 & 0 & 0 & 0 & 0 & 0 & 0 & 0 & 0 \\
\hline & 火 & 〈and〉 & 70 & 1 & 3 & 0 & 0 & 0 & 0 & 0 & 0 & 0 & 0 & 0 & 0 & 0 & 0 & 0 \\
\hline & 力 & ? & 0 & 28 & 0 & 0 & 0 & 0 & 0 & 0 & 0 & 0 & 0 & 0 & 0 & 0 & 0 & 0 \\
\hline \multirow{15}{*}{$\ddot{a}$} & 尚 ${ }_{023}$ & $\langle$ äd $\rangle$ & 0 & 0 & 10 & 0 & 2 & 0 & 0 & 0 & 0 & 0 & 0 & 0 & 0 & 0 & 0 & 0 \\
\hline & 业 062 & |ën〉 & 0 & 0 & 0 & 1 & 1 & 0 & 0 & 0 & 0 & 0 & 0 & 0 & 0 & 0 & 0 & 0 \\
\hline & 方 $_{073}$ & 〈än〉 & 0 & 0 & 4 & 0 & 107 & 16 & 0 & 0 & 0 & 0 & 0 & 0 & 0 & 0 & 0 & 0 \\
\hline & 有 084 & 〈är〉 & 0 & 0 & 42 & 0 & 44 & 4 & 0 & 0 & 0 & 0 & 0 & 0 & 0 & 0 & 0 & 0 \\
\hline & 方 093 & |ärd〉 & 0 & 0 & 0 & 0 & 2 & 0 & 0 & 0 & 0 & 0 & 0 & 0 & 0 & 0 & 0 & 0 \\
\hline & 为 168 & 〈äx〉 & 0 & 0 & 3 & 0 & 0 & 0 & 0 & 0 & 0 & 0 & 0 & 0 & 0 & 0 & 0 & 0 \\
\hline & 尓 ${ }_{197}$ & 〈äy〉 & 0 & 0 & 14 & 1 & 0 & 0 & 0 & 0 & 0 & 0 & 0 & 0 & 0 & 0 & 0 & 0 \\
\hline & 领 & 〈äm〉 & 0 & 0 & 22 & 2 & 27 & 0 & 0 & 0 & 0 & 0 & 0 & 0 & 0 & 0 & 0 & 0 \\
\hline & 幺 $_{274}$ & $\langle\ddot{a} \mathrm{~g}\rangle$ & 0 & 0 & 9 & 6 & 0 & 0 & 0 & 0 & 0 & 0 & 0 & 0 & 0 & 0 & 0 & 0 \\
\hline & 韭 280 & 〈äl〉 & 0 & 0 & 6 & 0 & 4 & 2 & 0 & 0 & 0 & 0 & 0 & 0 & 0 & 0 & 0 & 0 \\
\hline & 尚 299 & $?$ & 0 & 0 & 1 & 0 & 0 & 0 & 0 & 0 & 0 & 0 & 0 & 0 & 0 & 0 & 0 & 0 \\
\hline & 乐 $_{315}$ & 〈äš〉 & 0 & 0 & 0 & 23 & 0 & 0 & 0 & 0 & 0 & 0 & 0 & 0 & 0 & 0 & 0 & 0 \\
\hline & 文 $_{327}$ & 〈ëë〉 & 0 & 0 & 0 & 0 & 0 & 0 & 0 & 0 & 0 & 0 & 0 & 0 & 0 & 0 & 0 & 1 \\
\hline & 头 335 & |ää〉 & 0 & 0 & 0 & 4 & 0 & 0 & 0 & 0 & 0 & 0 & 0 & 0 & 0 & 0 & 0 & 0 \\
\hline & 生 359 & $?$ & 0 & 0 & 0 & 0 & 2 & 0 & 0 & 0 & 0 & 0 & 0 & 0 & 0 & 0 & 0 & 0 \\
\hline & 达 $_{115}$ & 〈öl〉 & 0 & 0 & 0 & 0 & 0 & 4 & 0 & 0 & 0 & 0 & 0 & 0 & 0 & 0 & 0 & 0 \\
\hline & 吅 ${ }_{324}$ & 〈ön〉 & 0 & 0 & 0 & 0 & 0 & 16 & 0 & 0 & 0 & 0 & 0 & 0 & 0 & 0 & 0 & 0 \\
\hline
\end{tabular}

(Continued on the next page) 


\begin{tabular}{|c|c|c|c|c|c|c|c|c|c|c|c|c|c|c|c|c|c|c|}
\hline \multirow{2}{*}{\multicolumn{3}{|c|}{$\overline{\mathrm{G}_{2}} \mathrm{G}_{1}$}} & \multicolumn{2}{|c|}{$a a$} & \multicolumn{2}{|c|}{$\ddot{a} \ddot{a}$} & \multirow{2}{*}{$\begin{array}{l}\ddot{e} \ddot{e} \\
\text { 文 } \\
\end{array}$} & \multirow{2}{*}{$\begin{array}{l}\ddot{O} \ddot{O} \\
\text { 芙 } \\
\end{array}$} & \multicolumn{2}{|c|}{$O O$} & \multicolumn{3}{|c|}{$u u / v \mho$} & \multirow{2}{*}{$\begin{array}{l}\ddot{u ̈ u} \\
\text { 岀 } \\
\end{array}$} & \multicolumn{2}{|c|}{$t$} & \multicolumn{2}{|c|}{$e e$} \\
\hline & & & 为 & $\mathrm{Caa}$ & 斗 & Cää & & & 及 & $\mathrm{CoO}$ & 及 & $\mathrm{Cuu}$ & 全 & & 兴 & $\mathrm{Cii}$ & 炎 & Cee \\
\hline \multirow{17}{*}{$O$} & 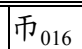 & 〈od〉 & 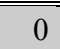 & & $\overline{0}$ & 0 & $\overline{0}$ & $\overline{0}$ & $\overline{77}$ & $\bar{~} 15$ & $\overline{0}$ & 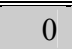 & $\bar{~} 0$ & $\overline{0}$ & 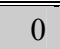 & $\bar{~} 0$ & $\overline{0}$ & $\overline{0}$ \\
\hline & 土L $_{076}$ & 〈or〉 & 0 & 0 & 0 & 0 & 0 & 0 & 119 & 51 & 0 & 0 & 0 & 0 & 0 & 0 & 0 & 0 \\
\hline & 太 & $\langle 0 y\rangle$ & 0 & 0 & 0 & 0 & 0 & 0 & 89 & 3 & 0 & 0 & 0 & 0 & 0 & 0 & 0 & 0 \\
\hline & 子 $_{149}$ & $\langle\mathrm{ol}\rangle$ & 0 & 0 & 0 & 0 & 0 & 0 & 208 & 45 & 0 & 0 & 0 & 0 & 0 & 0 & 0 & 0 \\
\hline & 小 $_{154}$ & $\langle o n\rangle$ & 0 & 0 & 0 & 0 & 0 & 0 & 11 & 17 & 0 & 0 & 0 & 0 & 0 & 0 & 0 & 0 \\
\hline & $\tau^{2}{ }_{157}$ & $\langle$ ols $\rangle$ & 0 & 0 & 0 & 0 & 0 & 0 & 24 & 1 & 0 & 0 & 0 & 0 & 0 & 0 & 0 & 0 \\
\hline & 欠 169 & $\left\langle\mathrm{OX}^{\mathrm{w}}\right\rangle$ & 0 & 0 & 0 & 0 & 0 & 0 & 0 & 1 & 0 & 1 & 0 & 0 & 0 & 0 & 0 & 0 \\
\hline & 及 ${ }_{186}$ & $\langle 00\rangle$ & 0 & 0 & 0 & 0 & 0 & 0 & 17 & 0 & 0 & 0 & 1 & 0 & 0 & 0 & 0 & 0 \\
\hline & 州 188 & $\langle 0 \mathrm{j}\rangle$ & 0 & 0 & 0 & 0 & 0 & 0 & 8 & 1 & 0 & 0 & 0 & 0 & 0 & 0 & 0 & 0 \\
\hline & 末 $_{217}$ & $\langle$ ond $\rangle$ & 0 & 0 & 0 & 0 & 0 & 0 & 1 & 90 & 0 & 0 & 0 & 0 & 0 & 0 & 0 & 0 \\
\hline & 行 ${ }_{220}$ & 〈om〉 & 0 & 0 & 0 & 0 & 0 & 0 & 0 & 31 & 0 & 0 & 0 & 0 & 0 & 0 & 0 & 0 \\
\hline & 众 ${ }_{252}$ & $\left\langle 0 \breve{g}^{\mathrm{w}}\right\rangle$ & 0 & 0 & 0 & 0 & 0 & 0 & 6 & 13 & 0 & 0 & 0 & 0 & 0 & 0 & 0 & 0 \\
\hline & 人 & 〈ol'〉 & 0 & 0 & 0 & 0 & 0 & 0 & 89 & 59 & 0 & 0 & 0 & 0 & 0 & 0 & 0 & 0 \\
\hline & 困 304 & 〈old〉 & 0 & 0 & 0 & 0 & 0 & 0 & 47 & 11 & 0 & 0 & 0 & 0 & 0 & 0 & 0 & 0 \\
\hline & 内 322 & 〈oň〉 & 0 & 0 & 0 & 0 & 0 & 0 & 122 & 0 & 0 & 0 & 0 & 0 & 0 & 0 & 0 & 0 \\
\hline & 尖 354 & $\langle 0 s\rangle$ & 0 & 0 & 0 & 0 & 0 & 0 & 29 & 5 & 0 & 0 & 0 & 0 & 0 & 0 & 0 & 0 \\
\hline & 米 ${ }_{355}$ & 〈ord〉 & 0 & 0 & 0 & 0 & 0 & 0 & 6 & 0 & 0 & 0 & 0 & 0 & 0 & 0 & 0 & 0 \\
\hline \multirow{20}{*}{$u$} & 米 $_{041}$ & |uUs〉 & 0 & 0 & 0 & 0 & 0 & 0 & 0 & 0 & 9 & 134 & 0 & 0 & 0 & 0 & 0 & 0 \\
\hline & 杏059 & 〈uň〉 & 0 & 0 & 0 & 0 & 0 & 0 & 0 & 0 & 11 & 0 & 0 & 0 & 0 & 0 & 0 & 0 \\
\hline & 井 $_{090}$ & $\left\langle\mho \breve{g}^{\mathrm{w}}\right\rangle$ & 0 & 0 & 0 & 0 & 0 & 0 & 1 & 0 & 0 & 1 & 0 & 0 & 0 & 0 & 0 & 0 \\
\hline & 交 097 & 〈ur〉 & 0 & 0 & 0 & 0 & 0 & 0 & 0 & 0 & 81 & 35 & 0 & 0 & 0 & 0 & 0 & 0 \\
\hline & 石 ${ }_{131}$ & 〈uu & 0 & 0 & 0 & 0 & 0 & 0 & 0 & 0 & 4 & 26 & 0 & 0 & 0 & 0 & 0 & 0 \\
\hline & $3_{148}$ & ? & 0 & 0 & 0 & 0 & 0 & 0 & 0 & 0 & 0 & 1 & 1 & 0 & 0 & 0 & 0 & 0 \\
\hline & 际 ${ }_{152}$ & $\langle u j ̌\rangle$ & 0 & 0 & 0 & 0 & 0 & 0 & 0 & 0 & 328 & 10 & 1 & 0 & 0 & 0 & 0 & 0 \\
\hline & 久 172 & $\left\langle\mathbf{u g}^{\mathbf{w}}\right\rangle$ & 0 & 0 & 0 & 0 & 0 & 0 & 0 & 0 & 14 & 6 & 0 & 0 & 0 & 0 & 0 & 0 \\
\hline & 补 ${ }_{177}$ & $\langle$ urǰ̀ & 0 & 0 & 0 & 0 & 0 & 0 & 0 & 0 & 0 & 35 & 0 & 0 & 0 & 0 & 0 & 0 \\
\hline & 化 $_{179}$ & 〈und & 0 & 0 & 0 & 0 & 0 & 0 & 0 & 0 & 18 & 2 & 6 & 6 & 0 & 0 & 0 & 0 \\
\hline & 矢 206 & $\left\langle u l^{\prime}\right\rangle$ & 0 & 0 & 0 & 0 & 0 & 0 & 0 & 0 & 6 & 2 & 0 & 0 & 0 & 0 & 0 & 0 \\
\hline & 化 236 & $\langle u d\rangle$ & 0 & 0 & 0 & 0 & 0 & 0 & 1 & 0 & 149 & 4 & 9 & 2 & 0 & 0 & 0 & 0 \\
\hline & 余 246 & $\left\langle U x^{w}\right\rangle$ & 0 & 0 & 0 & 0 & 0 & 0 & 0 & 0 & 0 & 2 & 1 & 0 & 0 & 0 & 0 & 0 \\
\hline & 分 249 & 〈urd & 0 & 0 & 0 & 0 & 0 & 0 & 0 & 0 & 0 & 0 & 46 & 0 & 0 & 0 & 0 & 0 \\
\hline & 炎 262 & 〈uy & 0 & 0 & 0 & 0 & 0 & 0 & 0 & 0 & 14 & 1 & 1 & 2 & 0 & 0 & 0 & 0 \\
\hline & 父273 & $\langle$ un $\rangle$ & 0 & 0 & 0 & 0 & 0 & 0 & 0 & 0 & 93 & 36 & 18 & 36 & 0 & 0 & 0 & 0 \\
\hline & $\mathrm{K}_{345}$ & $\left\langle\mathbf{u} \mathbf{w}^{\mathbf{w}}\right\rangle$ & 0 & 0 & 0 & 0 & 0 & 0 & 0 & 0 & 1 & 96 & 0 & 9 & 0 & 0 & 0 & 0 \\
\hline & 古 366 & $\langle u l\rangle$ & 0 & 0 & 0 & 0 & 0 & 0 & 0 & 0 & 76 & 17 & 0 & 0 & 0 & 0 & 0 & 0 \\
\hline & 八 $_{372}$ & $\left\langle u \breve{g}^{\mathrm{w}}\right\rangle$ & 0 & 0 & 0 & 0 & 0 & 0 & 0 & 0 & 0 & 155 & 0 & 0 & 0 & 0 & 0 & 0 \\
\hline & 半 & ? & 0 & 0 & 0 & 0 & 0 & 0 & 0 & 0 & 2 & 0 & 0 & 0 & 0 & 0 & 0 & 0 \\
\hline \multirow{2}{*}{$\ddot{u}$} & 爽 050 & $\langle$ üd & 0 & 0 & 0 & 0 & 0 & 0 & 0 & 0 & 0 & 0 & 0 & 10 & 0 & 0 & 0 & 0 \\
\hline & 不 & ? & 0 & 0 & 0 & 0 & 0 & 0 & 0 & 0 & 0 & 0 & 0 & 3 & 0 & 0 & 0 & 0 \\
\hline
\end{tabular}

(Continued on the next page) 


\begin{tabular}{|c|c|c|c|c|c|c|c|c|c|c|c|c|c|c|c|c|c|c|}
\hline \multirow{2}{*}{\multicolumn{3}{|c|}{$\mathrm{G}_{2} \mathrm{G}_{1}$}} & \multicolumn{2}{|c|}{$a a$} & \multicolumn{2}{|c|}{$\ddot{a} \ddot{a}$} & \multirow{2}{*}{\begin{tabular}{l|l|}
$\ddot{e} \ddot{e}$ \\
交 \\
\end{tabular}} & \multirow{2}{*}{$\begin{array}{ll}\ddot{O} 0 \\
\text { 莫 } \\
\end{array}$} & \multicolumn{2}{|c|}{$O O$} & \multicolumn{3}{|c|}{$u u / v \mho$} & \multirow{2}{*}{$\ddot{u ̈ u ̈}$} & \multicolumn{2}{|c|}{$i i$} & \multicolumn{2}{|c|}{$e e$} \\
\hline & & & 为 & $\mathrm{Caa}$ & 斗 & Cää & & & 及 & $\mathrm{CoO}$ & 交 & $\mathrm{Cuu}$ & 全 & & 兴 & Cii & & Cee \\
\hline \multirow{5}{*}{$\ddot{u}$} & $\nexists_{137}$ & |ür> & 0 & & 0 & 0 & 0 & 0 & 0 & 0 & 0 & 0 & 0 & 1 & 0 & 0 & 0 & 0 \\
\hline & 任 224 & 〈ül〉 & 0 & 0 & 0 & 0 & 0 & 0 & 0 & 0 & 0 & 0 & 0 & 2 & 0 & 0 & 0 & 0 \\
\hline & 非 282 & $\left\langle\ddot{\mathbf{u}} \mathbf{g}^{\mathbf{w}}\right\rangle$ & 0 & 0 & 0 & 0 & 0 & 0 & 0 & 0 & 0 & 0 & 0 & 5 & 0 & 0 & 0 & 0 \\
\hline & 岀 289 & 〈üǜ & 0 & 0 & 0 & 0 & 0 & 0 & 0 & 0 & 0 & 0 & 0 & 2 & 0 & 0 & 0 & 3 \\
\hline & 乃_ & ? & 0 & 0 & 0 & 0 & 0 & 0 & 0 & 0 & 0 & 0 & 0 & 5 & 0 & 0 & 0 & 0 \\
\hline \multirow{10}{*}{$i$} & 雨 018 & (in〉 & 0 & 0 & 0 & 0 & 0 & 0 & 0 & 0 & 0 & 0 & 0 & 0 & 37 & 31 & 0 & 0 \\
\hline & 忝 $_{033}$ & 〈išs $\rangle$ & 0 & 0 & 0 & 10 & 0 & 0 & 0 & 0 & 0 & 124 & 0 & 0 & 171 & 14 & 0 & 0 \\
\hline & 克 089 & $\langle$ id $\rangle$ & 0 & 0 & 0 & 0 & 4 & 0 & 0 & 0 & 0 & 0 & 0 & 0 & 4 & 0 & 0 & 0 \\
\hline & 又 $_{133}$ & $\langle i m\rangle$ & 0 & 0 & 0 & 0 & 0 & 0 & 0 & 0 & 0 & 0 & 0 & 0 & 9 & 0 & 0 & 0 \\
\hline & 勺 165 & $\langle i g\rangle$ & 0 & 0 & 0 & 0 & 0 & 0 & 0 & 0 & 0 & 0 & 0 & 0 & 29 & 1 & 0 & 0 \\
\hline & 化 235 & 〈ir & 0 & 0 & 0 & 0 & 1 & 0 & 0 & 0 & 0 & 0 & 0 & 0 & 254 & 1 & 0 & 0 \\
\hline & 用 302 & $\langle$ ill & 0 & 0 & 0 & 0 & 1 & 0 & 0 & 0 & 0 & 0 & 0 & 0 & 0 & 0 & 0 & 0 \\
\hline & 同 303 & (in) & 0 & 0 & 0 & 0 & 0 & 0 & 0 & 0 & 0 & 0 & 0 & 1 & 15 & 11 & 0 & 0 \\
\hline & 兴 339 & |iii & 0 & 0 & 0 & 0 & 0 & 0 & 0 & 0 & 0 & 0 & 0 & 0 & 13 & 1 & 0 & 0 \\
\hline & 㶢 352 & $\langle i \grave{g}\rangle$ & 0 & 0 & 0 & 0 & 0 & 0 & 0 & 0 & 0 & 0 & 0 & 0 & 0 & 10 & 0 & 0 \\
\hline \multirow{24}{*}{$e$} & 万̄ $_{020}$ & 〈ey〉 & 0 & 0 & 0 & 0 & 0 & 0 & 0 & 0 & 0 & 0 & 0 & 0 & 0 & 0 & 2 & 6 \\
\hline & 水 ${ }_{162}$ & $\langle$ ¿eǰ & 1 & 0 & 0 & 0 & 0 & 0 & 0 & 0 & 0 & 0 & 0 & 0 & 1 & 0 & 73 & 1 \\
\hline & 矢 205 & $\langle$ end $\rangle$ & 0 & 0 & 0 & 0 & 0 & 0 & 0 & 0 & 1 & 1 & 0 & 2 & 10 & 5 & 10 & 0 \\
\hline & 午 244 & 〈es〉 & 0 & 0 & 0 & 0 & 0 & 0 & 0 & 0 & 0 & 0 & 0 & 0 & 0 & 0 & 4 & 1 \\
\hline & 丹 $_{311}$ & $\langle e b\rangle$ & 0 & 0 & 0 & 0 & 1 & 0 & 0 & 0 & 0 & 0 & 0 & 0 & 0 & 0 & 8 & 0 \\
\hline & 火 $_{344}$ & $\langle$ 〈eld $\rangle$ & 0 & 0 & 0 & 0 & 0 & 0 & 4 & 0 & 37 & 1 & 0 & 0 & 1 & 0 & 14 & 0 \\
\hline & 枀 349 & $\langle e \breve{g}\rangle$ & 0 & 0 & 0 & 0 & 0 & 0 & 0 & 0 & 0 & 0 & 0 & 0 & 0 & 6 & 0 & 1 \\
\hline & 尘 356 & $\langle e l '\rangle$ & 0 & 0 & 0 & 0 & 0 & 0 & 0 & 0 & 0 & 0 & 1 & 3 & 1 & 0 & 20 & 5 \\
\hline & 比 $_{080}$ & 〈eel & 0 & 0 & 0 & 0 & 0 & 0 & 0 & 0 & 1 & 1 & 0 & 0 & 1 & 0 & 2 & 116 \\
\hline & 令 247 & $\langle$ eed $\rangle$ & 0 & 0 & 0 & 0 & 0 & 1 & 0 & 0 & 0 & 0 & 0 & 0 & 0 & 0 & 1 & 0 \\
\hline & 公 251 & $\langle$ een〉 & 0 & 0 & 0 & 0 & 0 & 2 & 0 & 0 & 0 & 0 & 0 & 0 & 0 & 0 & 5 & 0 \\
\hline & 企 257 & $\langle\mathrm{em}\rangle$ & 0 & 0 & 0 & 0 & 0 & 0 & 0 & 0 & 0 & 0 & 0 & 0 & 0 & 0 & 3 & 64 \\
\hline & 姿 341 & 〈eer & 1 & 0 & 0 & 0 & 12 & 0 & 1 & 0 & 5 & 1 & 1 & 0 & 0 & 0 & 7 & 4 \\
\hline & 当 361 & $\langle$ 〈eeň〉 & 0 & 0 & 0 & 0 & 0 & 0 & 0 & 0 & 6 & 0 & 0 & 0 & 1 & 0 & 0 & 6 \\
\hline & 共 ${ }_{108}$ & ? & 0 & 0 & 0 & 0 & 4 & 0 & 0 & 0 & 10 & 0 & 0 & 0 & 0 & 0 & 0 & 0 \\
\hline & 本 ${ }_{140}$ & $\langle e n\rangle$ & 0 & 0 & 0 & 0 & 0 & 0 & 0 & 0 & 0 & 1 & 0 & 1 & 21 & 16 & 4 & 0 \\
\hline & 及 $_{144}$ & |er $\rangle$ & 0 & 0 & 0 & 0 & 0 & 0 & 0 & 0 & 1 & 0 & 0 & 0 & 0 & 0 & 0 & 0 \\
\hline & 伏 222 & 〈eň〉 & 0 & 0 & 1 & 0 & 0 & 0 & 0 & 0 & 11 & 0 & 0 & 1 & 3 & 0 & 0 & 0 \\
\hline & 谷 254 & $\langle e d\rangle$ & 0 & 0 & 0 & 0 & 0 & 0 & 0 & 0 & 0 & 0 & 0 & 0 & 1 & 4 & 0 & 0 \\
\hline & 伞 ${ }_{258}$ & $\langle e z\rangle$ & 0 & 0 & 0 & 0 & 0 & 9 & 0 & 0 & 0 & 0 & 0 & 0 & 0 & 0 & 0 & 0 \\
\hline & 夾 ${ }_{261}$ & $\langle e l\rangle$ & 0 & 0 & 0 & 0 & 1 & 0 & 0 & 0 & 0 & 0 & 0 & 0 & 1 & 0 & 0 & 0 \\
\hline & 灸 264 & |en〉 & 0 & 0 & 0 & 0 & 0 & 0 & 0 & 0 & 0 & 2 & 0 & 1 & 2 & 1 & 0 & 0 \\
\hline & 朵 293 & 〈ens〉 & 0 & 0 & 0 & 0 & 5 & 0 & 0 & 0 & 13 & 0 & 0 & 0 & 3 & 0 & 0 & 0 \\
\hline & 炎 ${ }_{348}$ & (ee) & 0 & 0 & 0 & 0 & 0 & 6 & 0 & 0 & 1 & 0 & 0 & 0 & 0 & 0 & 0 & 0 \\
\hline- & 由 320 & 〈bel $\rangle$ & 1 & 0 & 0 & 0 & 0 & 0 & 0 & 0 & 0 & 0 & 0 & 0 & 0 & 1 & 0 & 0 \\
\hline
\end{tabular}


For instance, the grapheme 共 069 can follow the graphemes $\langle(\mathrm{C})$ aa $>$, but does not follow the other $(\mathrm{C}) \mathrm{V}$ graphemes. Similarly, all $\mathrm{G}_{2} \mathrm{~S}$ that can follow $\langle(\mathrm{C})$ aa $>\mathrm{s}$ do not follow the other $(\mathrm{C}) \mathrm{V}$ graphemes, though some exceptions were observed. This is true of the other vowels, too. ${ }^{12}$

Consequently, it appears that a $(\mathrm{C}) \mathrm{V}$ grapheme can only be followed by specific $\mathrm{G}_{2} \mathrm{~s}$. In order to interpret the data coherently, it is the most plausible explanation to presume that such $\mathrm{G}_{2} \mathrm{~S}$ are vowel-initial graphemes whose vowels are identical with the preceding one. Therefore, the following rule is inferred:

(ii) Vowel-overlapping rule: A vowel-final grapheme has to be followed by a vowel-initial grapheme whose vowel is identical with the preceding one.

This rule reveals that essentially all of the above-mentioned $\mathrm{G}_{2} \mathrm{~S}$ are $\mathrm{VC}$ graphemes, because the rule does not function between these graphemes and the following ones, and they are thus presumed as consonant-final graphemes. ${ }^{13}$ As such, the voweloverlapping rule is effectual in confirming the types and vocalic values of the graphemes.

This rule is applied in principle whenever a grapheme is spelled after a vowelfinal grapheme (e.g. 丈为共 〈eš-aa-al’〉 šaal’ ${ }^{15}$, but if the most suitable VC grapheme does not exist in the KSS syllabary, then the second most suitable one is spelled, such

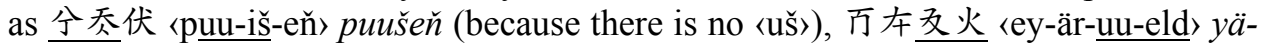
ruuld (no 〈uld〉), 个文哭〈es-uu-ens〉suuns (no〈uns〉). In addition, there were relatively many spellings that slightly deviated from the rule. However, they mostly had another spelling according to the rule and are fewer than the regular ones, hence they were assumed to be "non-orthographic". 16

${ }^{12}$ There is no space to discuss the individual exceptions here. As for the main factor of exceptions, see the description below in this section.

${ }^{13}$ This consequence is supported by an analysis of Khitan transcriptions of Chinese words discussed in Section 2.3. Exceptional sequences $\langle(\mathrm{C}) \mathrm{VV}-\mathrm{VV}\rangle$ are occasionally observed, most of which are optional and peculiar spellings exclusive to Chinese qusheng 去聲 syllables (Shen 2012).

${ }^{14}$ Some researchers have reconstructed VCV graphemes such as 杏 〈uni〉, 坴〈ali〉, 生〈abu〉 from the inadequate evidence as stated in Section 1. The vowel-overlapping rule proves that such reconstructions are incorrect and they are VC graphemes. Furthermore, many researchers have reconstructed * (335)'s value as a diphthong 〈ia〉. However, viewed from the vowel-overlapping phenomenon, the characteristic of the grapheme is similar to that of 文 〈ëë〉 rather than 为〈aa〉. We thus have to reconstruct the value of $\mathcal{F}$ as a vowel whose quality is similar to that of $\ddot{e}$ rather than $a$.

${ }^{15}$ A similar rule is applied after semivowel-final graphemes, such as 火灸亿-〈ek-uy-ir〉 $k u y r$-, 灸用〈uy-il〉 uyl, 土早〈ew-ul〉 ewl, 介交 〈xaw-ur〉xawr. The grapheme 用 (302) 〈il〉 is not attested in the position after $\langle(\mathrm{C}) \mathrm{ii}\rangle$ graphemes in my corpus, but it is often followed by $\langle(\mathrm{C}) \mathrm{Vy}\rangle$ graphemes, thus the value is presumed to be $\langle\mathrm{iC}\rangle$.

${ }^{16}$ For example, 亢斗乃 〈eg-ää-am〉gääm (3 examples) vs. 亢斗圣 〈eg-ää-äm〉 gääm (22 examples), and 不忝 〈xää-iš̀ xä̈äs (7 examples) vs. 不办 〈xää-äšs xääs (19 examples). 


\section{Reconstructing the Khitan Vowel System}

Based on the reconstructed phonetic values, I will use this section to establish the regular vowel correspondences between Khitan and Mongolian in addition to describing the phonological changes that explain these correspondences.

Firstly, I added another $\mathrm{V}$ grapheme to the twelve graphemes shown in Table 2. See the following example of rhyming words in an epitaphic rhyme:

汉炎 keer, 伸令 $\ddot{\theta} \ddot{\theta} r$, 水炎 čeer,

罗炎 $C V V r$, 万交炎 yëer , 午专炎 $s \underline{V V} r(N u u$ 43-45)

The grapheme ${ }^{7}{ }_{125}$, which is not used for Chinese words, rhymes with the vowels $e, \ddot{e}$, and $\ddot{\theta}$ in closed-syllable words. Thus, it must represent a vowel whose quality is similar to those of $e, \ddot{e}$, and $\ddot{\theta}$. I reconstructed its value as ${ }^{*} \theta$ based on a correspondence to be discussed in Section 5.2.

\subsection{Vowel Length}

Vowels represented by V or CV graphemes are long. This is because long vowels in the modern Mongolian languages such as Mongolian (Mong.), ${ }^{17}$ Kalmyk (Kalm.), and Dagur (Dag.) or hiatuses in Middle Mongolian (MMo.) correspond to Khitan (Khit.) vowels represented by $\mathrm{V}$ or $\mathrm{CV}$ graphemes: ${ }^{18}$

\begin{tabular}{|c|c|c|c|c|c|c|}
\hline \multicolumn{4}{|c|}{ Khit. } & Meaning & MMo. & Mong. \\
\hline \multirow{13}{*}{$\overline{\text { (a) }}$} & 巫 & 〈xaа〉 & $x a a$ & 'khaghan' & $x a^{\prime} a n$ & $x a a \eta$ \\
\hline & 企为 & 〈äm-aa〉 & $\ddot{a m a} \underline{a}$ & 'sheep' & ima'an & yamaa \\
\hline & 子- & $\langle$ j̆aa-〉 & jaa- & 'to tell' & $j i{ }^{\prime} a-$ & jaa- \\
\hline & 令力为 & 〈et-äx-aa〉 & täxaa & 'chicken' & takiya & $\overline{d a ̈ x} a a$ \\
\hline & 丈电为 & 〈eš-aw-aa〉 & šawa & 'falcon' & šiba'un & $\check{s} \sigma b \overline{\sigma v}$ \\
\hline & 丹有为乎 & 〈eb-är-aa-an〉 & bäraan & 'right-hand' & bara'un & baruvon \\
\hline & 爸斗 & 〈ed-ää〉 & $d \underline{a ̈ a}$ & 'enemy' & dayin & $d \underline{a ̈ a ̈} \eta$ \\
\hline & 专矢为 & 〈taw-ul'-aa〉 & tawl'a & 'hare, rabbit' & taula & tovlääa \\
\hline & 汉 & 〈kee-〉 & kee- & 'to say' & ke'e- & - \\
\hline & 东炎 & $\langle$ eč-ee〉 & jee $e^{19}$ & 'sister's child' & ji'e & jee \\
\hline & 及劵 & $\langle\overline{\mathrm{er}}-\mathrm{ee}\rangle$ & eree & 'now' & $\overline{e d o ̈ ' e}$ & Dag. edee \\
\hline & 雨 $\overline{\text { 子 }}$ & 〈doo-ol-〉 & dogl- & 'to hear' & $d \overline{u^{\prime} u l}-$ & dovl- \\
\hline & $\bar{\imath}$ & $\langle\mathrm{kuu}\rangle$ & kuu & 'person' & $k \overline{\ddot{u} \prime u ̈ n}$ & Kalm. küün \\
\hline
\end{tabular}

${ }^{17}$ Mongolian mentioned henceforward refers to the Chakhar dialect in Inner Mongolia.

${ }^{18}$ Mongolian long vowels derived from MMo. hiatuses $A^{\prime} U$ in initial syllables, however, correspond to Khitan diphthongs $a w$ and ew, e.g. 防 jaw 'hundred' || MMo. $\breve{j a}$ 'un, Mong. 方 dew 'man's younger sibling of the same sex' $\|$ MMo. de'ü, Mong. duu 'younger brother'. See Ōtake (2016b, p. 24).

${ }^{19}$ This word means “one's sister's child” and “one's daughter's child” (Ōtake 2016c). 
Some Khitan long vowels were derived from vowels in monosyllabic opensyllable words. It appears to be the traces of the minimal word condition which is a constraint that prohibits the occurrence of monomoraic words.

\begin{tabular}{|c|c|c|c|c|c|c|}
\hline & Khit. & & & Meaning & MMo. & Mong. \\
\hline \multirow{5}{*}{ (b) } & 为- & $\langle a a-\rangle$ & $\underline{a a}-$ & 'to be' & $\underline{a}-$ & $\underline{a}-\sim \underline{a a}-$ \\
\hline & 㶫 & $\langle e e\rangle$ & $\underline{e e}$ & 'this' & $\underline{e}(n e)$ & $\underline{e} n$ \\
\hline & 承 & $\langle$ poo $\rangle$ & poo & 'time, season' & $h \underline{o}(n)$ & $\underline{o \eta}$ \\
\hline & 全 & $\langle\mho \mho\rangle$ & $\underline{U U}$ & 'water' & $\underline{u}(\operatorname{sun})$ & $\underline{v s}$ \\
\hline & 丹专- & $\langle e b-\Theta \theta-\rangle$ & $b \underline{\theta \theta}^{-}$ & 'to be' & $b \underline{\ddot{g}}-$ & - \\
\hline
\end{tabular}

More interestingly, long vowels in early loanwords correspond to Turkic primary long vowels that are mainly reconstructed by data from modern languages like Yakut (Yak.) and Turkmen. It implies that Proto-Mongolic (PMo. ${ }^{20}$ distinguished between vowel lengths, which was preserved in Khitan. Such primary long vowels are also observed in Khitan native words as shown in Section 5.2. ${ }^{21}$

\begin{tabular}{|c|c|c|c|c|c|c|c|}
\hline & Khit. & & & Meaning & MMo. & OTü. & Yak. \\
\hline (c) & $\begin{array}{l}\text { 丹及子- } \\
\text { 几卡 } \\
\text { 米及 }\end{array}$ & $\begin{array}{l}\text { 〈eb-oo-ol-〉 } \\
\langle\text { kuu-us〉 } \\
\langle\text { ord-uu〉 }\end{array}$ & $\begin{array}{l}\text { bool- } \\
\text { kuus }^{22} \\
\text { orduu }\end{array}$ & $\begin{array}{l}\text { 'to become' } \\
\text { 'strength' } \\
\text { 'ordo' }\end{array}$ & $\begin{array}{l}\text { bol- } \\
\text { küčün } \\
\text { ordo }\end{array}$ & $\begin{array}{l}\text { bol- } \\
k \ddot{u} c ̌ \\
o r d u\end{array}$ & $\begin{array}{l}b \underline{u o} l-<* b \bar{o} l- \\
k \underline{\ddot{u} u} s \\
\text { ord } \underline{u u}\end{array}$ \\
\hline
\end{tabular}

\subsection{Regular Vowel Correspondences}

In principle, the Mongolian unrounded short vowels $a, e, i$, or the MMo. $a, e, i$, respectively, correspond to the Khitan short vowels $a, e, i$ (d) or the long vowels $a a$, $e e, i i(\mathrm{e})$ :

\begin{tabular}{|c|c|c|c|c|c|c|}
\hline & Khit. & & & Meaning & MMo. & Mong. \\
\hline \multirow[t]{6}{*}{ (d) } & 丹冬 & 〈eb-as〉 & bas & 'again, also' & basa & $b \underline{a s}$ \\
\hline & 百旭 & 〈ey-aw-〉 & $y \underline{a} w-$ & 'to conduct' & yábu- & $y \underline{a} b-$ \\
\hline & 午木 & 〈es-en〉 & esen & 'healthy' & esen & esen \\
\hline & 八夾 & 〈ek-el〉 & ke $l$ & 'word' & kelen & $x \underline{e} l$ \\
\hline & 忝 & $\langle i \check{S}\rangle$ & $\underline{i s}$ & 'nine' (fem.) & yisiün & $y \underline{i s}$ \\
\hline & 조 & 〈jir〉 & $\bar{j} \underline{i} r^{23}$ & 'two' (fem.) & jürin & - \\
\hline \multirow[t]{3}{*}{ (e) } & 为- & $\langle\mathrm{aa}-\rangle$ & $\underline{a a}-$ & 'to be' & $\underline{a}-$ & $\underline{a}-\sim \underline{a a}-$ \\
\hline & 炎 & 〈ee〉 & $\underline{\overline{e e}}$ & 'this' & $\underline{\underline{e}}(n e)$ & $\underline{\underline{e} n}$ \\
\hline & 水兴忝 & 〈eč-ii-išs & $\bar{c} \underline{i} \underline{i} \underline{s}$ & 'blood relative' & čisun & jos \\
\hline
\end{tabular}

${ }^{20}$ I employ the term "Proto-Mongolic" as the hypothetical common ancestor of all attested Mongolic languages including so-called "Para-Mongolic" (Janhunen 2003).

${ }^{21}$ For a more detailed discussion on Khitan vowel length, see Ōtake $(2015 \mathrm{c})$.

${ }^{22}$ To decipher this word, see Ōtake (2015a).

${ }^{23}$ This word rhymes with the syllables Cir, such as 亿 ir and 令亿 tir. 
The Mongolian rounded short vowel $o$, or the MMo. $o$, corresponds to the Khitan short $o$ or long $o$ (f); the Mongolian short $v$, or the MMo. $u$, corresponds to the Khitan short $o$ or long $v(\mathrm{~g})$. This reveals that the merger of the Proto-Mongolic short $* u$ with the short ${ }^{*} o$ took place, but the long * $u$ was retained as the long $v$ in Khitan.

\begin{tabular}{|c|c|c|c|c|c|c|}
\hline & Khit. & & & Meaning & MMo. & Mong. \\
\hline \multirow[t]{3}{*}{ (f) } & 米文 & 〈ord-uu〉 & orduu & 'ordo' & ordo & ord \\
\hline & 丹及子- & 〈eb-oo-ol-〉 & bool- & 'to become' & $\bar{b} \underline{o} l-$ & $\bar{b} \underline{o} l-$ \\
\hline & 再圠 & $\langle$ doo-or〉 & door & 'under' & doro & dor $\sim$ door \\
\hline \multirow[t]{4}{*}{ (g) } & 尔 & $\langle$ 〈on-〉 & & 'to fall, drop' & $\underline{u} n a-$ & $\underline{v} n-$ \\
\hline & 再子- & $\langle$ doo-ol-〉 & dool- ${ }^{24}$ & 'to hear' & $\bar{d} \underline{u}^{\prime} \underline{u} l-$ & $\bar{d} \underline{v v l}-$ \\
\hline & 全 & 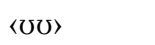 & $\underline{\bar{U}}$ & 'water' & $\underline{u}($ sun $)$ & $\underline{U S}$ \\
\hline & 马公 & 〈juu-un〉 & $\overline{\bar{y} \underline{\sigma \sigma} n^{25}}$ & 'summer' & $\bar{\jmath} \underline{u} n$ & $\bar{\jmath} \underline{\underline{v}} \eta$ \\
\hline
\end{tabular}

Similarly, the Mongolian rounded short vowel $u$, or the MMo. $\ddot{u}$, corresponds to the Khitan short $u$ or long $u$ (h); the Mongolian short $\theta$, or the MMo. $\ddot{o}$, corresponds to the Khitan short $u$ or long $\theta$ (i). This shows that the merger of the ProtoMongolic short * $\ddot{o}$ with the short $* \ddot{u}$ occurred, but the long $* \ddot{o}$ was preserved as the long $\theta$ in Khitan.

\begin{tabular}{|c|c|c|c|c|c|c|}
\hline & Khitan & & & Meaning & MMo. & Mong. \\
\hline \multirow[t]{3}{*}{$\overline{(h)}$} & 业早- & 〈ep-ul-〉 & $p \underline{u} l-$ & 'to exceed' & hüle- & $\underline{u} l-$ \\
\hline & 及早 & $\langle$ uu-ul $\rangle$ & $\underline{u u l}$ & 'winter' & $\underline{u} b \underline{u} l$ & $\bar{\theta} b \theta l^{26}$ \\
\hline & 几止 & 〈kuu-us〉 & kuus & 'strength' & küucü̈n & $x \underline{u} \check{c}$ \\
\hline (i) & 羊杏 & 〈es-uň〉 & $s \underline{u \underline{n}}$ & 'night' & sönni & $\check{s} \underline{\theta} n \sim s \underline{\theta} n$ \\
\hline & 分- & $\left\langle u \breve{g}^{w}-\right\rangle$ & $\bar{u}^{w^{w}}$ & 'to give' & $\ddot{o g}-$ & $\underline{\theta g}-$ \\
\hline & $\begin{array}{l}\text { 丹责- } \\
\text { 军 }\end{array}$ & $\begin{array}{l}\langle\mathrm{eb}-\theta \theta-\rangle \\
\langle\mathrm{d} \theta \Theta \mathrm{r}\rangle\end{array}$ & 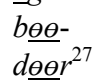 & $\begin{array}{l}\text { 'to be' } \\
\text { 'four' (fem.) }\end{array}$ & 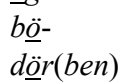 & $\overline{d \underline{\theta}} r \theta b$ \\
\hline
\end{tabular}

The phonemic changes concerning vowels that occurred between Proto-Mongolic and Khitan are summarised in Table $9 .{ }^{28}$

${ }^{24}$ This example shows that the merger of $* u(\sigma)$ with $*_{o}$ precedes the contraction of hiatuses *V'V > VV (i.e. PMo. *duğul- $>*$ do'ol- $>$ Khit, dool-).

${ }^{25}$ In order to coherently explain the relevant sound changes, I assumed that the graphemes with a phonetic value «Cuu〉 can represent both /Cuu/ and /Cvo/.

${ }^{26}$ The correspondence between the MMo. übül and the Mong. $\theta b \theta l$ is irregular.

${ }^{27}$ This word rhymes with the syllables Ceer, Cөөr, and Cëër. The dative-locative form of

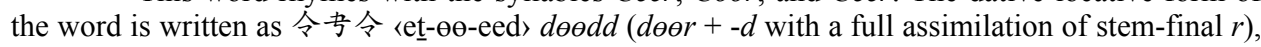

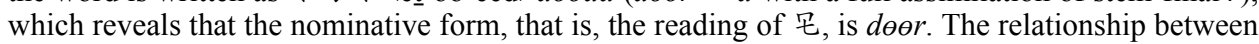
the Khit. deer and the Proto-Turkic *tört 'four' is unknown.

${ }^{28}$ For convenience, I treated vowels in monosyllabic open-syllable words equally to primary long vowels in this table, though the former are phonologically short in the PMo. stage. 
Table 9

\begin{tabular}{|c|c|c|c|}
\hline PMo. & Khit. & PMo. & Khit. \\
\hline$* a$ & $a$ & $* \bar{a}$ & $a a$ \\
\hline$*_{e}$ & $e$ & $* \bar{e}$ & $e e$ \\
\hline$*_{i}$ & $i$ & $*_{\bar{l}}$ & $i i$ \\
\hline$*_{o}$ & & $* \bar{o}$ & $o o$ \\
\hline$* u$ & $O$ & $* \bar{u}$ & $U U$ \\
\hline$* \ddot{o}$ & & $* \bar{o}$ & $\theta \theta$ \\
\hline$* \ddot{u}$ & $u$ & $* \bar{u}$ & $u u$ \\
\hline
\end{tabular}

\subsection{Palatalised Vowels}

Khitan has a series of palatalised vowel phonemes (i.e. $\ddot{a}, \ddot{e}, \ddot{o}, \ddot{\theta}, \ddot{u}$ ), which seem to be palatalised versions of plain vowels $a, e, o, \theta, u$, respectively. Phonological alternations without a semantic change are found in the following examples:

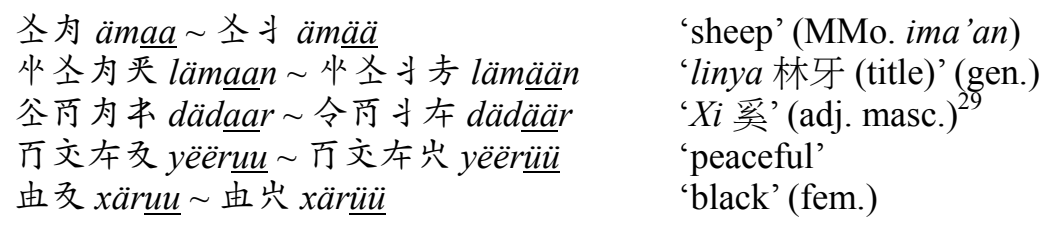

These synchronic free variations show that the progressive assimilation of a vocalic feature frontness is in progress in Khitan. As such, frontness is neutralised in non-initial syllables, but still functions in initial syllables.

The short palatalised vowel $\ddot{a}$ is derived from $*_{i}$ before $* a$ or $* a$ before $*_{i}(\mathrm{j}){ }^{30}$ the long $\ddot{a}$ is derived from $*$ ayi $(\mathrm{k})$. The origin of the other palatalised vowels is unclear.

\begin{tabular}{|c|c|c|c|c|c|c|}
\hline & Khit. & & & Meaning & MMo. & OTü. \\
\hline \multirow[t]{3}{*}{ (j) } & 企为 & 〈äm-aa〉 & $\underline{\ddot{a} m a a}$ & 'sheep' & ima'an & 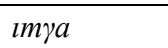 \\
\hline & 乎 & 〈mäy〉 & $m \ddot{a} \eta^{31}$ & 'thousand' & minqan & biy, biy \\
\hline & 令力为 & 〈et-äx-aa〉 & täxaa & 'chicken' & takiya & taqıүu \\
\hline \multirow[t]{2}{*}{ (k) } & 空斗 & 〈ed-ää〉 & $\overline{d a ̈ a}$ & 'enemy' & dayin & $y a \gamma l<* \delta a \gamma l$ \\
\hline & 丈斗 & 〈eš-ää〉 & $\check{s} \underline{a ̈ a}$ & 'good' & sayin & say \\
\hline
\end{tabular}

${ }^{29}$ This ethnic group is transcribed as 迭達 (ZY *dyä.da) in the Liaoshi (Ōtake 2016a, p. 10).

${ }^{30}$ However, if the onset is (alveo-)palatal $\check{c}, \breve{J}, \check{s}$ or $y$, the vowel $*_{i}$ resulted in $a$, not $\ddot{a}$, such as in 丈必为 (eš-aw-aa) šawaa 'falcon' (transcribed as 稍瓦 ZY *šaw.wa in the Liaoshi) \| MMo. šiba'un 'id.'; 肎为 〈čal-aa) čalaa 'stone' (transcribed as 查剌 ZY *ča.la in the Liaoshi as a person's name) || MMo. čila'un 'id.'.

${ }^{31}$ This word is also used to represent the Chinese 孟 (LMC *máy) (Ōtake 2015b, p. 8). 
"Irregular" correspondences concerning palatalised vowels are frequently observed as shown in (1), which may shed a light on the origin of Khitan palatalised vowels.

\begin{tabular}{|c|c|c|c|c|c|}
\hline \multicolumn{4}{|c|}{ Khit. } & Meaning & MMo. \\
\hline (l) & 丹有为觅 & 〈eb-är-aa-an〉 & bäraan <*bira'un? & 'right-hand' & bara'un \\
\hline & 丹力 & 〈eb-äx〉 & $b \underline{a} x<*$ biga? & 'child' & ba $\gamma a$ \\
\hline & 天 & 〈ňär〉 & $\check{n} \underline{\ddot{a}} r^{32}<*$ nira $a(n) ?$ & 'sun, day' & naran \\
\hline & 伏力 & 〈eň-äx〉 & $\check{n} \underline{\ddot{a}} x<* n \underline{i} k a(n) ?$ & 'dog' & nóqai \\
\hline & 达 & 〈öl〉 & $\underline{\ddot{o}}<* i \underline{i} l o(n) ?$ & 'much, many' & olon \\
\hline & 不亿 & $\langle e m-i r\rangle$ & $\operatorname{mi} r<* \operatorname{mir} i(n) ?$ & 'horse' & morin \\
\hline
\end{tabular}

\section{Conclusion}

Through the above discussion, the twelve vowel phonemes listed in Table 10 have been reconstructed in Khitan. ${ }^{33}$

\section{Table $10^{34}$}

\begin{tabular}{lccccccccccccc}
\hline & \multicolumn{1}{c}{ Plain vowels } & \multicolumn{1}{c}{ Palatalised vowels } \\
& $a$ & $e$ & $i$ & $o$ & $U$ & $\theta$ & $u$ & $\ddot{a}$ & $\ddot{e}$ & $\ddot{o}$ & $\ddot{\theta}$ & $\ddot{u}$ \\
\hline [Open] & + & + & - & + & - & + & - & + & + & + & + & - \\
[Front] & - & - & + & - & - & - & - & + & + & + & + & + \\
{$[$ Round $]$} & - & - & - & + & + & + & + & - & - & + & + & + \\
{$[$ RTR $]$} & + & - & - & + & + & - & - & + & - & + & - & - \\
\hline
\end{tabular}

Khitan vowel phonemes are distinguished by four vocalic features. These features [open], [front] and [round], respectively, explain the distinctions between nonhigh vowels vs. high ones, front vs. non-front (back), and rounded vs. unrounded. The feature [RTR] (retracted tongue root) is needed to explain the harmonic phenomena which were not mentioned in this paper. In addition, vowel length is also distinctive as stated earlier.

\section{References}

Janhunen, Juha (2003): Para-Mongolic. In: Janhunen, Juha (ed.): The Mongolic Languages. London, Routledge, pp. 391-402.

Kane, Daniel (2009): The Kitan Language and Script. Leiden, Brill (HdO VIII, vol. 19).

${ }^{32}$ This word is transcribed as 捏離 $(\mathrm{ZY} *$ *yä.li) in the Qidan guozhi 契丹國志. (It has been miscopied as 担離 $\mathrm{ZY} *$ dan.li in the Yanbei zaji 燕北雜記 collected in the Leishuo 類說.) It rhymes with the syllables $C \ddot{r} r$ or $C \ddot{r} r$, such as 公布 när and 由 $x \ddot{a} r$.

${ }^{33}$ This excludes the non-native phoneme $\imath$ that is used for Chinese loanwords.

34 "+” means the presence of the feature; "-" indicates its absence. 
Ōtake Masami 大竹昌巳 (2015a): Kittango no hōši hyōgen 契丹語の奉仕表現. KOTONOHA Vol. 149 , pp. $1-15$.

Ōtake Masami (2015b): Kittan šōji bunken šoin no kanbun kotenseki 契丹小字文獻所引の漢文古 典籍. KOTONOHA Vol. 152, pp. 1-19.

Ōtake Masami (2015c): Kittan šōi bunken ni okeru boin no nagasa no kakiwake 契丹小字文獻に おける母音の長さの書き分け. Gengo Kenkyū 言語研究 Vol. 148, pp. 81-102.

Ōtake Masami (2016a): Kittan šōji bunken ni okeru 'shixuan zhi jia' 契丹小字文獻におけ る「世選之家」. KOTONOHA Vol. 159, pp. 1-12.

Ōtake Masami (2016b): Kittan šǒji bunken ni okeru ‘boin kan no g’ 契丹小字文獻における「母 音間のg」. Nihon Mongoru gakkai kiyō日本モンゴル學會紀要 Vol. 46, pp. 19-34.

Ōtake Masami (2016c): Kittan šōji Yelü Wotela bošimē šoken no kōtē gō wa Tenso kōtē ni arazu 契丹小字『耶律斡特剌墓誌銘』所見の皇帝號は天祚皇帝に非ず．KOTONOHA Vol. 161, pp. 1-19.

Qingge'ertai 清格爾泰 et al. (1985): Qidan xiaozi yanjiu 契丹小字研究. Beijing 北京, Zhongguo shehui kexue chubanshe 中國社會科學出版社.

Shen Zhongwei 沈鍾偉 (2006): Liaodai beifang Hanyu fangyan de yuyin tezheng 遼代北方漢語 方言的語音特徵. Zhongguo yuwen 中國語文 No. 6, pp. 483-498.

Shen Zhongwei (2007): Sino-Khitan Phonology. Bulletin of Chinese Linguistics Vol. 1, No. 2, pp. $147-210$.

Shen Zhongwei (2009): Qidan xiaozi yunwen chutan 契丹小字韻文初探. Minzu yuwen 民族語文 No. 3, pp. 37-50.

Shen Zhongwei (2012): Qidan xiaozi Hanyu yinyi zhong de yi ge shengdiao xianxiang 契丹小字漢 語音譯中的一個聲調現象. Minzu yuwen No. 1, pp. 39-50. 